\title{
Mutation of 4-coumarate: coenzyme A ligase 1 gene affects lignin biosynthesis and increases the cell wall digestibility in maize brown midrib5 mutants
}

\author{
Wangdan Xiong ${ }^{1 \dagger}$, Zhenying Wu ${ }^{1 \dagger}$, Yuchen Liu' ${ }^{1}, Y u \mathrm{Li}^{1}$, Kunlong Su${ }^{1}$, Zetao Bai', Siyi Guo², Zhubing Hu², \\ Zhiming Zhang ${ }^{3}$, Yan Bao ${ }^{1}$, Juan Sun ${ }^{4}$, Guofeng Yang ${ }^{4}$ and Chunxiang Fu ${ }^{1 *}$ (D)
}

\begin{abstract}
Background: Maize brown midrib $(\mathrm{bm})$ mutants associated with impaired lignin biosynthesis are a potential source for the breed of novel germplasms with improved cell wall digestibility. The spontaneous bm5 mutants had been identified since 2008. However, the gene responsible for the bm5 locus, and the comprehensive effects of bm5 mutation on lignin biosynthesis, soluble phenolics accumulation, and cell wall degradation have yet to be elucidated.

Results: The bm5 locus was identified to encode a major 4-coumarate: coenzyme A ligase (Zm4CL1) through analyzing MutMap-assisted gene mapping data. Two alleles of $Z m 4 C L 1$ isolated from bm5 mutants contained two transposons inserted in the first exon and the second intron, respectively, and consequently, the activities of $4 \mathrm{CLs}$ in the crude enzyme extracts from bm5 midribs were reduced by 51-62\% compared with the wild type. Furthermore, five $4 C L s$ were retrieved from maize genome, and $Z m 4 C L 1$ was the most highly expressed one in the lignified tissues. Mutation of Zm4CL1 mainly impeded the biosynthesis of guaiacyl (G) lignins and increased the level of soluble feruloyl derivatives without impacting maize growth and development. Moreover, both neutral detergent fiber digestibility and saccharification efficiency of cell walls were significantly elevated in the bm5 mutant.

Conclusions: Zm4CL1 was identified as the Bm5 gene, since two independent alleles of Zm4CL1 were associated with the same mutant phenotype. Mutation of $Z m 4 C L 1$ mainly affected $\mathrm{G}$ lignin biosynthesis and soluble feruloyl derivatives accumulation in maize lignified tissues. The reduced recalcitrance of the $b m 5$ mutant suggests that Zm4CL1 is an elite target for cell wall engineering, and genetic manipulation of this gene will facilitate the utilization of crop straw and stover that have to be dealt with for environmental protection.
\end{abstract}

Keywords: Maize, brown midrib, Cell wall digestibility, Lignin, 4-coumarate: coenzyme A ligase

\footnotetext{
*Correspondence: fucx@qibebt.ac.cn

†Wangdan Xiong and Zhenying Wu have contributed equally to this work

${ }^{1}$ Key Laboratory of Biofuels, Shandong Provincial Key Laboratory of Energy Genetics, Qingdao Institute of Bioenergy and Bioprocess Technology, Chinese Academy of Sciences, Qingdao 266101, China Full list of author information is available at the end of the article
} 


\section{Introduction}

Plant cell walls are structural supporter and natural barrier in protecting the plants from pathogens and insects, which consist mainly of cellulose, hemicellulose, and lignin [1]. Lignin is a complex and heterogeneous aromatic polymer that is an important component for structural support, water transport, and biotic and abiotic stress defenses during plant growth and development [2]. The biosynthesis of monolignols generates from the general phenylpropanoid metabolism, following by a series of hydroxylation and methylation reactions [2]. It has been reported that lignin content and its subunit composition are closely related to the bioconversion efficiency of the lignocellulosic biomass into fermentable sugars [1, 3]. Moreover, the presence of lignin in cell walls also negatively impacts forage digestibility and pulping efficiency [4].

4-coumarate: coenzyme A ligase (4CL) is an essential enzyme in lignin biosynthetic pathway, converting $p$-coumarate, caffeate, and ferulate into their corresponding CoA esters $[2,5]$. The $4 \mathrm{CL}$ family is a small and conserved gene family, playing a critical role in the biosynthesis of phenylpropanoid metabolites [6]. The 4CLs can be divided into two major subgroups: one group is mainly responsible for lignin biosynthesis; the other group is involved in flavonoid biosynthesis [6]. In Arabidopsis, three $4 C L$ isoforms, namely At4CL1, At4CL2, and At4CL4, participate in lignin biosynthesis, and At4CL3 are responsible for flavonoid metabolism [7]. Moreover, five $4 C L$ isoforms have been characterized in rice and each isoform shows different substrate affinities in vitro [8]. Suppression of Os4CL3 in rice leads to lignin reduction and impacts plant development and growth [8]. Similarly, downregulation of Pv4CL1 and Sh4CL1 reduces lignin content in switchgrass and sugarcane [9-11]. Sitedirected mutagenesis of Pp4CL1 characterizes the function of conserved amino acids, providing an insight in understanding the relationship between structure and function of 4CL proteins in Peucedanum praeruptorum [12].

The spontaneous brown midrib (bm or bmr) mutants in maize, sorghum, and pearl millet exhibit brownish midribs, associated with impaired lignin biosynthesis [13]. To date, at least six maize $b m$ mutations ( $b m 1-b m 6)$ have been identified and four of them have been characterized [14-17]. The loci of maize $b m 1$ and $b m 3$ encode the cinnamyl alcohol dehydrogenase (CAD) and caffeoyl$O$-methyltransferase (COMT), respectively, which are crucial enzymes in the lignin biosynthetic pathway [14, 17], whereas the loci of $b m 2$ and $b m 4$ are responsible for the biosynthesis of methyl donor required for the biosynthesis of both guaiacyl (G) and syringyl (S) monolignols $[15,16]$. Moreover, two sorghum $b m r$ loci have been identified including CAD (bmr6) and COMT (bmr12 and bmr18) [13]. Recently, sorghum Bmr2 has been identified to encode a major 4CL [18]. The corresponding $\mathrm{bm}$ mutant in maize, however, has yet to be found. The $b m$ mutants associated with dramatic changes in lignin content and/or composition are of great interest to improve agro-industrial properties of corn stover. For example, a typical successful case was the commercial utilization of $b m 3$ mutant that has a high forage digestibility [13]. Thus, the characterization of novel $b m$ mutations will shade light on the molecular mechanism of lignin biosynthesis, which may lead to produce more valuable sources for breeding new germplasm of forage and biofuel crops with high cell wall digestibility.

The maize $b m 5$ mutants had been previously identified early in 2008 [19]. The $b m 5$ locus was roughly mapped to chromosome 5 , in the same bin as $b m 1[19,20]$. The chemical analysis further revealed that $b m 5$ mutation reduced the levels of Klason lignin, G monomers, and wall-bound $p$-coumarate, but increased the levels of $\mathrm{H}$ monomers and wall-bound ferulate in maize mature stems [21]. However, the $b m 5$ gene has yet to be identified, and the comprehensive effects of $b m 5$ mutation on lignin biosynthesis, soluble phenolics accumulation, and cell wall degradation are still elusive. In this study, we identified $Z m 4 C L 1$ as the $b m 5$ gene and its mutation impeded $\mathrm{G}$ lignin biosynthesis and, consequently, increased the level of feruloyl quinic acid (FQA) and feruloyl glycoside (FG) that are derived from ferulate, an intermediate in the lignin biosynthetic pathway in maize. Moreover, the maize mutants exhibited a normal growth and development. Finally, the disruption of lignin biosynthesis resulted in a significant increase in dry matter forage digestibility and saccharification efficiency of cell walls.

\section{Results}

\section{Morphological characterization of $b m 5$ mutants}

Three inbred stocks of $b m 5$ mutant were obtained from the Maize Genetic Cooperation Stock Center, designated as 504I, 504J, and 505J. The bm5 mutants exhibited normal growth and development in our greenhouse condition except the typical reddish-brown pigmentation in the midrib and stalk compared with the wild type collected from 60 days old (Fig. 1a-f). The cross sections of midribs from $b m 5$ mutant and wild-type plants further revealed that the pigmentation mainly deposited in sclerenchyma tissues (Fig. 1g, h). Moreover, the above cross sections were stained with the phloroglucinol- $\mathrm{HCl}$ reagent. The reduced staining area of sclerenchyma tissues indicates a less lignin accumulation in the $b m 5$ mutant (Fig. 1i, j). 

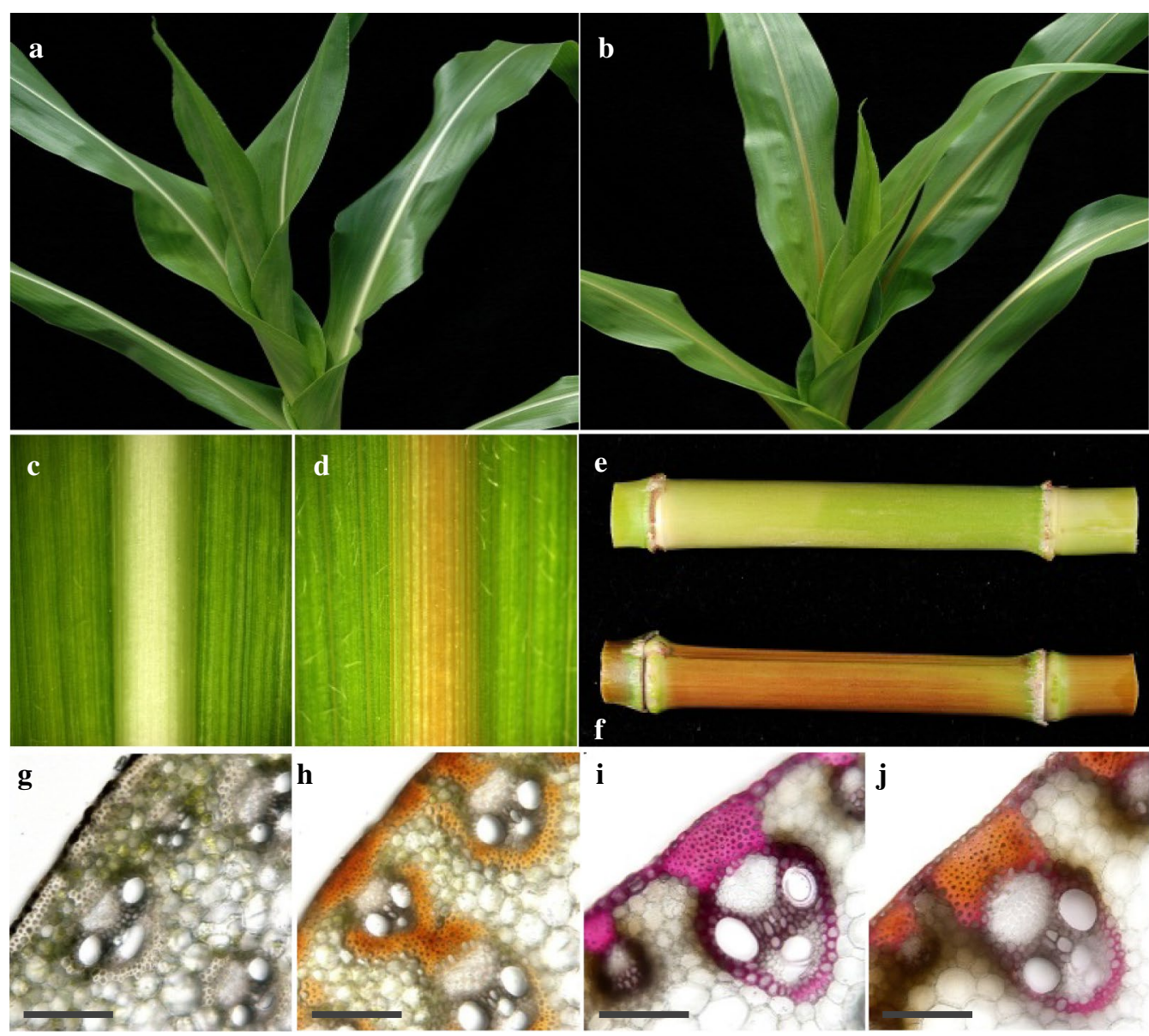

Fig. 1 Morphological characterization of the bm5 mutants. a Greenhouse grown B73 wild-type maize. b Greenhouse grown bm5 mutant. c Adaxial view of the representative midrib of the fourth leaf collected from 60-day-old B73 wild-type maize. $\mathbf{d}$ Adaxial view of the representative midrib of the fourth leaf collected from 60-day-old bm5 mutant. e The fourth internode collected from 60-day-old B73 wild-type maize. f The fourth internode collected from 60-day-old bm5 mutant. $\mathbf{g}$ The midrib cross section from the fourth leaf collected from 60-day-old B73 wild-type maize without staining. $\mathrm{Bar}=1.0 \mathrm{~mm}$. $\mathbf{h}$ The midrib cross section from the fourth leaf collected from 60 -day-old $b \mathrm{~m} 5 \mathrm{mutant}$. Bar $=1.0 \mathrm{~mm}$. i Phloroglucinol- $\mathrm{HCl}$ staining of the midrib cross section from the fourth leaf collected from 60-day-old B73 wild-type maize. Bar $=1.0 \mathrm{~mm}$. $\mathbf{j}$ Phloroglucinol- $\mathrm{HCl}$ staining of the midrib cross section from the fourth leaf collected from 60-day-old bm5 mutant

\section{Identification of the $b m 5$ gene}

The $b m 5$ locus is an independent $b m$ allele and has been mapped to maize chromosome 5 in the bin 5.04 region $(80.8-172.4 \mathrm{Mb})[19,20]$. The $b m 5$ locus was further mapped to be linked with p-umc1591 in bin 5.04 region using simple sequence repeat (SSR) markers published in MaizeGDB database (Additional file 1: Table S1). To identify the $b m 5$ gene, a MutMap-assisted gene mapping based on the whole-genome sequencing technology was employed to narrow down the $b m 5$ locus. The maize stock $b m 5-504 \mathrm{~J}$ was directly crossed with $\mathrm{B} 73$ to generate F1 individuals, and then, the F1 individual was selfed to produce F2 progeny. DNA mixture pool from about 96 F2 individuals with brown midrib phenotype was sequenced by Illumina sequencing with depth of more than $10 \times$ coverage. By analyzing SNPindex results, the mutation region was narrowed to 80.8-120.7 Mb region on chromosome 5, and 1302 genes were in this region (Fig. 2a; Additional file 2: Table S2). Among them, only two lignin biosynthesis genes were retrieved. One is cinnamyl alcohol dehydrogenase (CAD2, GRMZM5G844562), the same gene as $B M 1$ [14]; the other candidate gene is Zm4CL1 (GRMZM2G075333) encoding a 4-coumarate: coenzyme A ligase (Additional file 2: Table S2). Given that $b m 1$ and $b m 5$ were independent $b m$ alleles, we suspect that $Z m 4 C L 1$ rather than $C A D 2$ was the most considerable $b m 5$ gene (Fig. 2b). To confirm our hypothesis, the full-length genomic sequences of $Z m 4 C L 1$ alleles including $5^{\prime}$ untranslated region, six exons, five introns, and $3^{\prime}$ untranslated region 


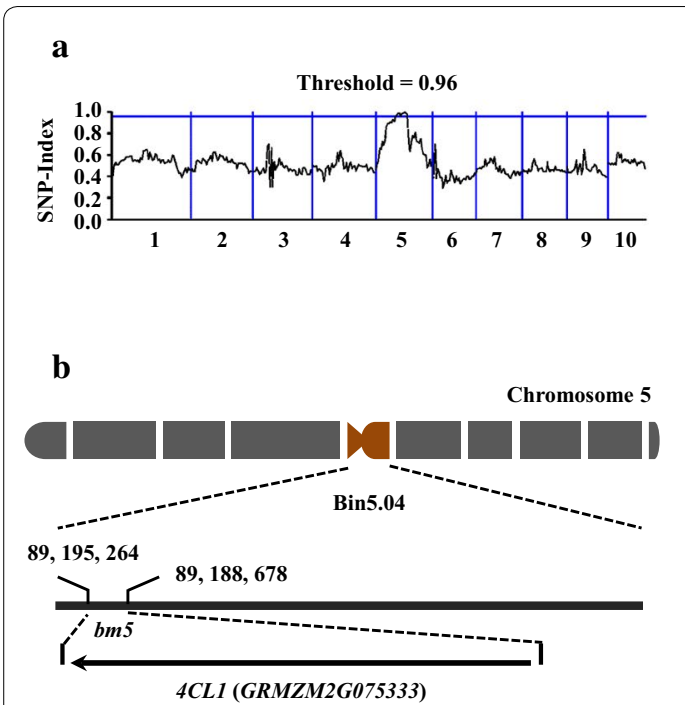

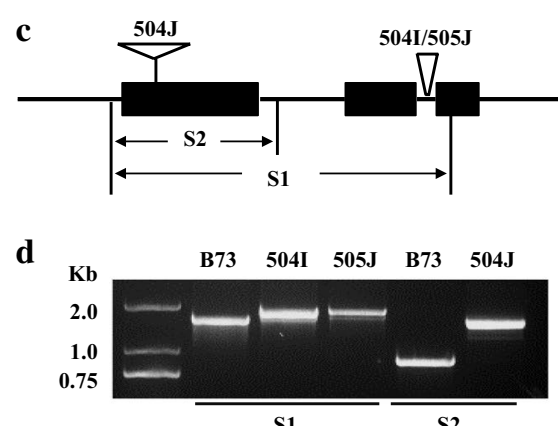

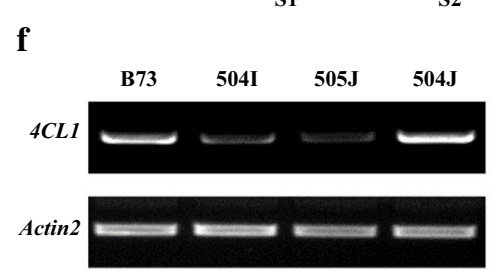

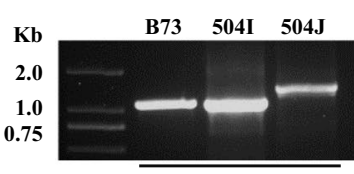

S1

Fig. 2 Identification of the bm5 gene. a The SNP-index for the F2 progeny of bm5-504J. b Genomic location of Zm4CL 1 (GRMZM2G075333) on maize chromosome 5. The base pair number was referred to the Phytozome database version 1.8. c Exon and intron structure of the $Z \mathrm{~m} 4 \mathrm{CL} 1 \mathrm{gene}$ Black boxes indicate exons and lines between the boxes indicate introns. Insertion sequences of bm5 are indicated by inverse triangles. $\mathbf{d}$ S1 PCR products amplified from B73, bm5-504l, and bm5-505J genomic DNA and S2 PCR products amplified from B73 and bm5-504J genomic DNA. e S1 PCR products amplified from B73, bm5-504l, and bm5-504J CDNA. f RT-PCR analysis of Zm4CL 1 expression in midribs of B73 wild-type and bm5 mutants. $\mathbf{g}$ qRT-PCR analysis of Zm4CL 1 expression in midribs of B73 wild-type and bm5 mutants. The primer pair was designed in $3^{\prime}$ untranslated region. Midribs of the fourth leaves were collected from 60-day-old B73 wild-type and bm5 mutants

were obtained from $b m 5$ mutants and B73 by polymerase chain reaction (PCR) amplification and subsequent sequencing. Sequence alignment and PCR analysis revealed a $283 \mathrm{bp} \mathrm{Mu}$ insertion in the second intron of $Z m 4 C L 1$ in the bm5-504I mutant (Fig. 2c, d; Additional file 3: Fig. S1). Moreover, the same mutation of the $Z m 4 C L 1$ gene was identified in bm5-505J mutant, implying the same original $b m 5$ allele (Fig. 2c, d; Additional file 3: Fig. S1). Furthermore, reverse transcription polymerase chain reaction (RT-PCR) analysis showed that the $\mathrm{Mu}$ insertion in the second intron was properly spliced off in the transcripts during their maturation (Fig. 2e). In addition, a $658 \mathrm{bp}$ Ac insertion in the first exon of bm5-504J allele resulted in a premature stop codon in the transcribed $Z m 4 C L 1$, even if the mRNA was produced (Fig. 2c-e; Additional file 3: Fig. S1). Furthermore, the molecular markers designed based on the two insertion sequences were employed to determine the relationship between mutation sites and mutant phenotype (Additional file 4: Table S3). The result showed that the two independent mutation sites in Zm4CL1 alleles were exactly associated with the same $b m$ phenotype, suggesting that $Z m 4 C L 1$ is the $b m 5$ gene.

\section{Impacts of the insertions on $\mathrm{Zm4CL} 1$ function}

The bm5 near-isogenic lines (NILs) in the B73 background were generated after six backcrosses of 504I and
504J with B73, respectively. To study the impacts of the insertions on $Z m 4 C L 1$ function, we first determined the expression level of Zm4CL1 alleles in bm5 mutants and B73. Both RT-PCR and quantitative RT-PCR (qRT-PCR) analyses revealed that the expression level of Zm4CL1 in both bm5-504I and bm5-505J mutants was dramatically reduced compared with B73 (Fig. 2f, g). However, no difference was observed between the expression level of $Z m 4 C L 1$ in $b m 5-504 J$ mutant and B73 (Fig. 2f, g). Furthermore, two truncated proteins were predicted and expressed in $E$. coli, since the Ac insertion caused a premature stop codon in $Z m 4 C L 1$ in $b m 5-504 J$ mutant. The soluble protein extracts containing the truncated Zm4CL1 mutants, Zm4CL1-S (95 amino acids) and Zm4CL1-L (469 amino acids), did not exhibit a 4CL enzyme activity (Fig. 3, Additional file 5: Fig. S2).

Furthermore, the soluble crude plant proteins were extracted from the midribs of bm5-NIL mutants and B73 wild-type plants. The activities of extracted proteins were analyzed using $p$-coumarate, caffeate, and ferulate substrates. The results revealed a dramatic reduction in 4CL enzyme activities of the extractable crude proteins from $b m 5$ mutants compared with B73 wild-type plants (Fig. 4). The 4CL activities for all the used substrates were reduced by $51-62 \%$ in $b m 5-504 \mathrm{I}$ and $b m 5-504 \mathrm{~J}$ mutants (Fig. 4). 
a

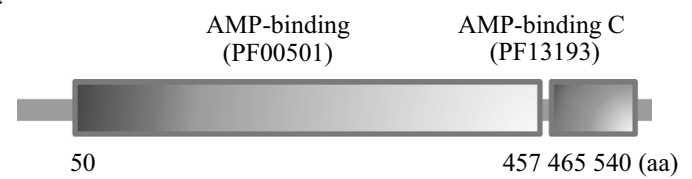

b

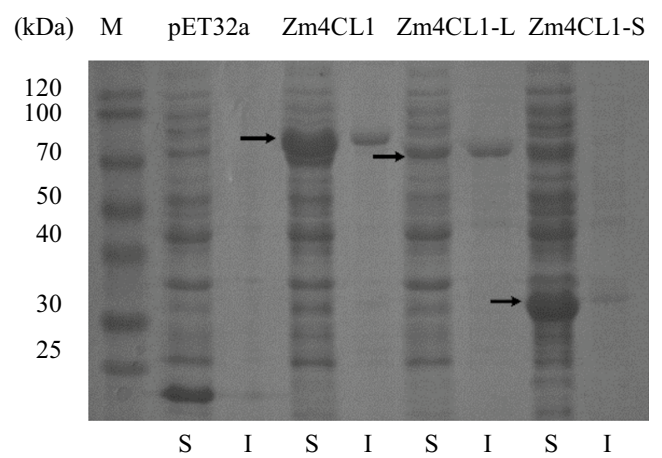

c

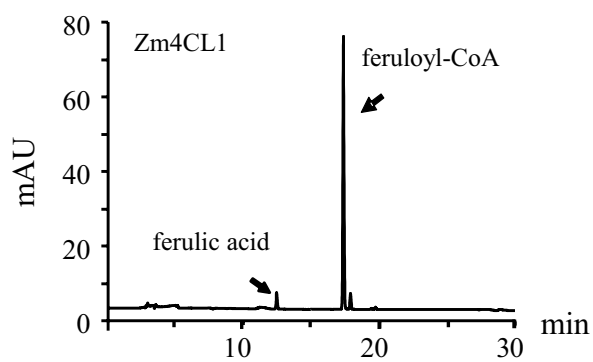

d

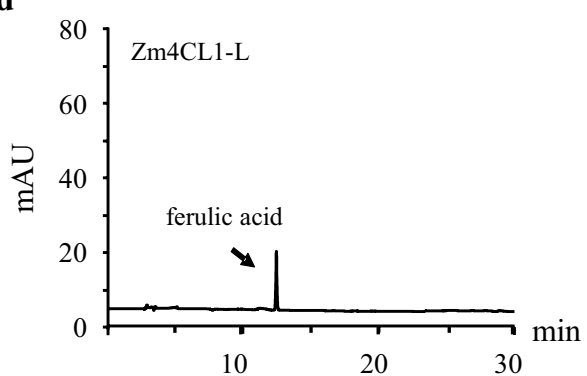

Fig. 3 The $4 C L$ activity of putative versions of Zm4CL1 isolated from bm5-504J mutant. a Two domains of Zm4CL1 were labeled. Image was generated using open-source SMART (http://smart.embl-heidelberg.de/). b SDS-PAGE analysis of recombinant Zm4CL1, Zm4CL1-L, and Zm4CL1-S proteins. $S$ and I indicated soluble and insoluble proteins from lysate of $E$. coli cultures. The arrows showed the recombinant proteins. $\mathbf{c} H P L C$ analysis of recombinant Zm4CL1 activity against ferulate. $\mathbf{d}$ HPLC analysis of recombinant Zm4CL1-L activity against ferulate

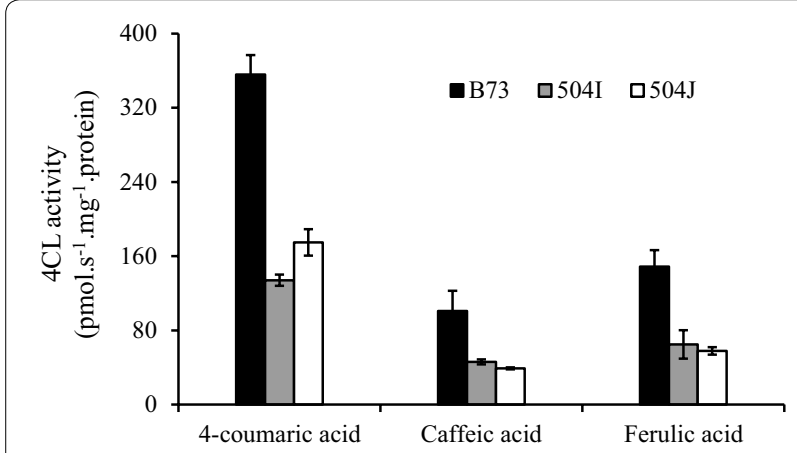

Fig. 4 Effect of Zm4CL1 mutations on 4CL enzyme activities. Crude enzyme extracts were prepared from the midribs of B73, bm5-504I, and bm5-504J. The 4CL enzyme activities of the above crude enzyme extracts against $p$-coumarate, caffeate, and ferulate were determined. Midribs of the fourth leaves were collected from 60-day-old B73 wild-type and bm5 mutants

\section{Phylogenetic and expression pattern analysis of $Z m 4 C L$ genes}

The $4 C L$ family consists of a cluster of small and conserved genes in plants, playing a crucial role for phenylpropanoid biosynthesis. Five $Z m 4 C L s$ were retrieved in maize genome, and a phylogenetic tree was built using 4CL deduced amino acid sequences from six species. 4CLs were clustered into two main groups, groups
I and II, and the members from the dicots and monocots were gathered separately in each subgroup (Additional file 6: Fig. S3). Zm4CL1 belongs to group I and is closely clustered with rice Os4CL3 (LOC_Os02g08100) and sorghum BMR2 (Sb04g005210), implying a conserved function in lignin biosynthesis (Additional file 6: Fig. S3). Furthermore, the RNA sequencing data were downloaded from the MaizeGDB database and analyzed, which revealed a higher expression level of $Z m 4 C L 1$ in all tested vegetative tissues compared with the other $Z m 4 C L$ genes, particularly in well-lignified root and internode tissues (Additional file 7: Table S4).

\section{Effects of $Z m 4 C L 1$ mutation on lignin biosynthesis}

We assessed the global effects of Zm4CL1 mutation on lignin biosynthesis by Affymetrix microarray analysis. Compared with B73 wild-type plant, a total of 57 transcripts, many of which are involved in the process of carbohydrate metabolism, cellular metabolites, primary metabolites, and macromolecule metabolites, were differentially expressed in $b m 5-504 \mathrm{~J}$ mutant (Additional file 8: Fig. S4, Additional file 9: Table S5). However, none of genes were involved in monolignol biosynthesis, implying that the disruption of $Z m 4 C L 1$ did not trigger the expression of other genes related to lignin biosynthesis in the mutant except its paralogs (Additional file 10: Table S6). The expression level of $Z m 4 C L 1$ paralog, 
GRMZM2G174732, was increased in both bm5-504I and bm5-504J mutants. In contrast, the expression levels of other Zm4CL1 paralogs including GRMZM2G054013, GRMZM2G048522, and GRMZM2G055320 were only increased in the $b m 5-504 \mathrm{~J}$ mutant, but did not exhibit a similar change in the $b m 5$-504I mutant (Additional file 11: Fig. S5).

We next examined lignin content and composition to investigate the effects of $Z m 4 C L 1$ mutation on lignin biosynthesis. Extractive free cell wall residues (CWRs) were employed to analyze the total lignin content and composition. Our results revealed a similar amount of total lignin between $b m 5$-504J mutant and B73 wild-type plant as measured by the acetyl bromide $(\mathrm{AcBr})$ method (Additional file 12: Table S7). Lignin composition analysis further showed that $\mathrm{G}$ lignin was dramatically reduced in $b m 5-504 \mathrm{~J}$, which only amounted to $42.3 \%$ of the control (Additional file 12: Table S7). The content of S lignin, however, has no significant change, as a consequence, the S/G ratio raised dramatically from 0.58 in $\mathrm{B} 73$ to 1.47 in bm5-504J (Additional file 12: Table S7). In contrast, the yield of $\mathrm{H}$ lignin was increased in the mutant (Additional file 12: Table S7). In addition, the relative percentage of $\mathrm{H}$ and $\mathrm{S}$ lignins were remarkably increased from $4.3 \%$ $(\mathrm{H} / \mathrm{H}+\mathrm{G}+\mathrm{S})$ and $35.1 \%(\mathrm{~S} / \mathrm{H}+\mathrm{G}+\mathrm{S})$ in $\mathrm{B} 73$ to $7.8 \%$ and $54.9 \%$ in the mutant, whereas $\mathrm{G}$ lignin still remained at a low level (37.3\% in $b m 5$ versus $60.5 \%$ in B73) (Additional file 12: Table S7).

\section{Soluble phenolic characterization of bm5 mutants}

To gain the effects of lignin disruption on its intermediates, the soluble phenolics were extracted from midribs and characterized by soluble phenylpropanoid profiling through reversed-phase liquid chromatography coupled with photo-diode array detection and electrospray ionization tandem mass spectrometry (LC-PDA-ESI-MS/ MS). An approximate 2.7-5.0-fold increase in FQA (peak 1) was revealed in bm5-504I and bm5-504J mutants (Fig. 5a-d). Most importantly, we identified one novel metabolite (peak 2) present in the mutant, but absent in B73 (Fig. 5a). The analysis of UV-visible and mass spectra further showed that the peak 2 was the glucoside derivative of ferulate (Fig. 5e, f, Additional file 13: Table S8). Furthermore, the compound of peak 2 was confirmed to be FG by comparing with the standard FG produced by AtUGT84A1 toward ferulate and UDP-glucose using LC-PDA-ESI-MS/MS (Additional file 13: Table S8, Additional file 14: Fig. S6, Additional file 15: Fig. S7b, Additional file 16: Fig. S8a-c). Moreover, the AtUGT84A1 homologous genes, ZmUGT84A-1 (GRMZM2G417945) and ZmUGT84A-2 (GRMZM2G304712), were isolated from maize and expressed in E. coli. As expected, the soluble protein extracts containing ZmUGT84A-1 and
ZmUGT84A-2 converted ferulate and UDP-glucose into FG in vitro (Additional files 15: Fig. S7c, d, 16: Fig. S8a-c). To gain an insight on the possible biosynthetic pathway of FQA in maize, we added FG into the crude extractive protein of maize midribs from $b m 5-504 \mathrm{~J}$ mutant and B73 wild-type plant. As expected, the crude extractive proteins from $b m 5-504 \mathrm{~J}$ mutant and B73 plant had capacity to catalyze the formation of FQA (Fig. 6, Additional file 16: Fig. S8d, f, Additional file 17: Fig. S9).

\section{Disruption of Zm4CL1 improved cell wall digestibility}

Given that lignin is a crucial factor negatively impacting lignocellulosic biomass utilization, we evaluated the effects of the altered lignin biosynthesis resulted from the bm 5 mutation by a fast and nondestructive near infrared reflectance spectroscopy (NIRS) method. Disruption of $Z m 4 C L 1$ led to a significant reduction in acid detergent lignin (ADL), whereas it had no effects on cellulose and hemicellulose accumulation (Additional file 18: Table S9). To study if the dramatically altered lignin biosynthesis could improve forage digestibility of the $b m 5$ mutants, neutral detergent fiber digestibility (NDFD) frequently employed for evaluating or marketing forages was evaluated. Our result revealed a substantially improved forage digestibility (i.e., a relative increase of $22.0 \%$ ) in the mutants compared with B73 wild-type plants (Fig. 7a). In addition, we also studied the effect of lignin alteration on the degradation efficiency of cell wall polysaccharides. Our result showed that the saccharification efficiency was increased from $51.0 \%$ in $B 73$ to $60.0 \%$ in the $b m 5$ mutant (i.e., a relative increase of $17.6 \%$ ) (Fig. 7b).

\section{Discussion}

The spontaneous bm5 mutation was identified in 2008 and roughly mapped to maize chromosome $5[19,21,22]$. In our work, the $b m 5$ mutant gene $Z m 4 C L 1$ was identified to encode a 4-coumarate: coenzyme A ligase through a MutMap-assisted gene mapping based on the wholegenome sequencing technology. Mutation of Zm4CL1 resulted in a typical brown midrib phenotype accompanied by the alteration of lignin and soluble phenolics in $b m 5$ mutants. Moreover, the disruption of lignin biosynthesis led to a significant improvement in both forage digestibility and saccharification efficiency of cell wall polysaccharides. Thus, our results suggest that Zm4CL1 is a potential target for cell wall engineering, and the $b m 5$ mutants are interest sources that could be integrated into breeding for development of novel germplasm with a high cell wall conversion rate.

The expression level of $Z m 4 C L 1$ was relatively higher than the other four $4 C L$ genes, especially in the welllignified tissues of B73 wild-type plants. The phylogenetic tree analysis shows that $\mathrm{Zm} 4 \mathrm{CL} 1$ is clustered with 


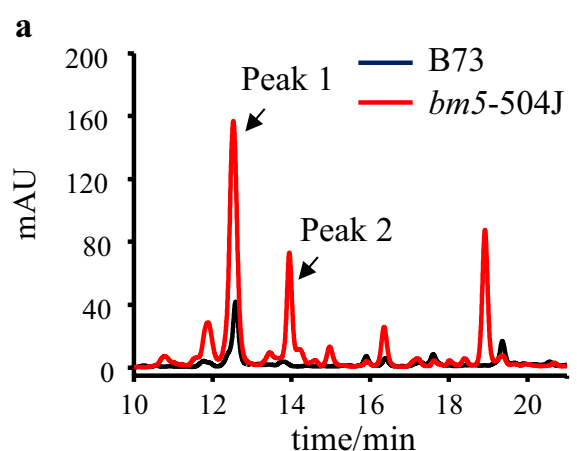

c

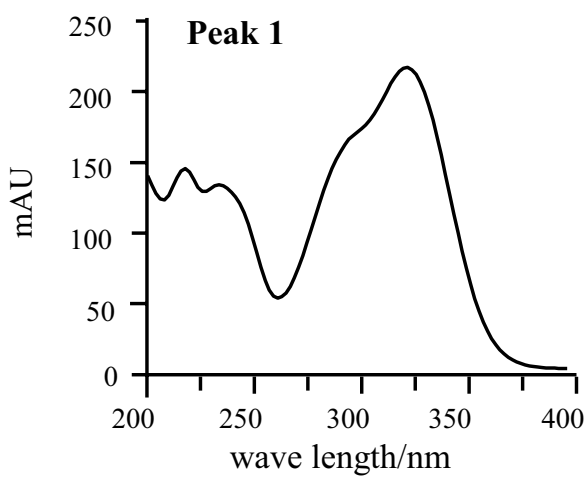

e

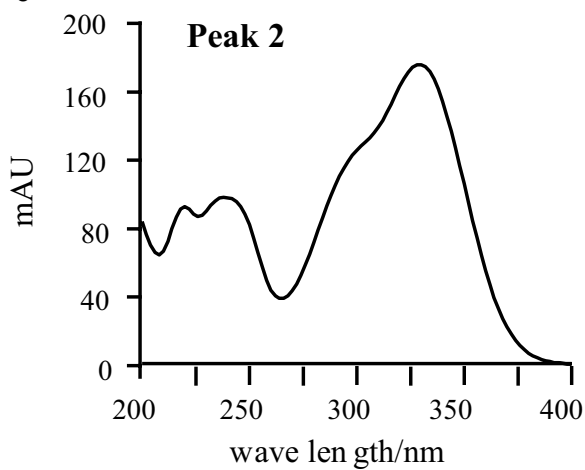

b

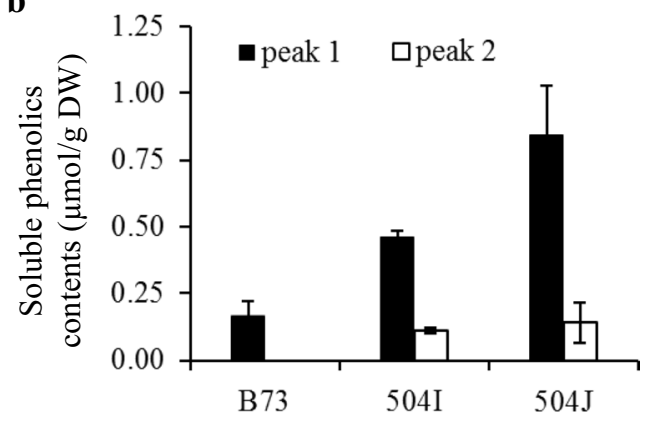

d

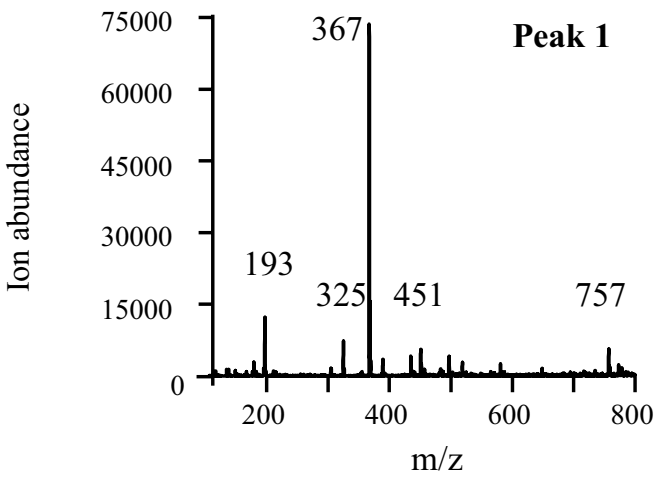

f

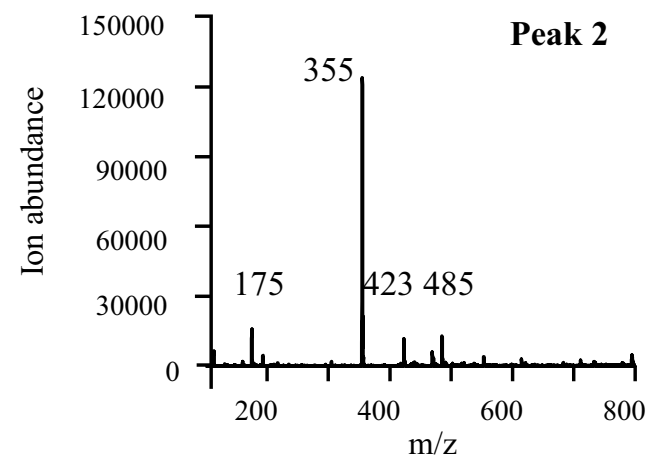

Fig. 5 Effects of Zm4CL1 mutations on phenylpropanoid accumulation in bm5 mutants. a The profile of soluble phenolics in methanolic extracts from midribs of bm5-504J mutant and B73 wild-type maize. The profile of soluble phenolics was performed by LC-PDA-ESI-MS/MS. b The contents of FQA (peak 1) and FG (peak 2) in bm5 mutants and B73 wild-type maize. Midribs of the second-to-fifth leaves from the top were collected from 60-day-old B73 wild-type maize, bm5-504I and bm5-504J mutants. Values are mean $\pm S E(n=3)$. DW, dry weight. FQA, feruloyl quinic acid. FG, feruloyl glucoside. c-fThe remarkably accumulated phenolics in the bm5 mutant were preliminarily identified as FQA (peak 1) and FG (peak 2) based on their UV-visible spectra (c and $\mathbf{e}$ ) and mass spectra ( $\mathbf{d}$ and $\mathbf{f}$ )

BMR2, Os4CL3, and Pv4CL1, which are the major genes responsible for lignin biosynthesis of sorghum, rice, and switchgrass $[8,11,18]$. Taken together, our results imply that $Z m 4 C L 1$ was a major isoform involved in lignin biosynthesis in maize. Moreover, two types of insertion mutations were found in the three bm5 mutants. One is $\mathrm{Mu}$ transposon insertion in the second intron of
Zm4CL1 of bm5-504I and bm5-505J, which had potential to reduce the expression levels of $Z m 4 C L 1$ dramatically and, consequently, reduced the $4 \mathrm{CL}$ activity in maize. The previous studies have suggested that introns can significantly regulate gene expression in many direct and indirect ways in plants [23]. It is not surprising that the $\mathrm{Mu}$ insertion in the intron of Zm4CL1 of bm5-504I 

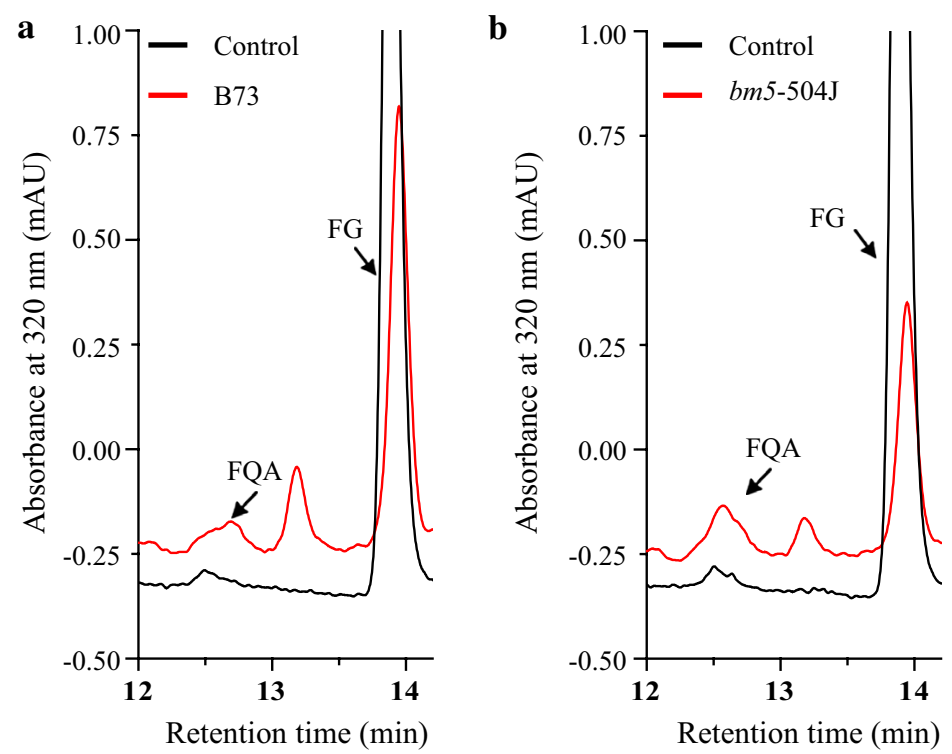

c
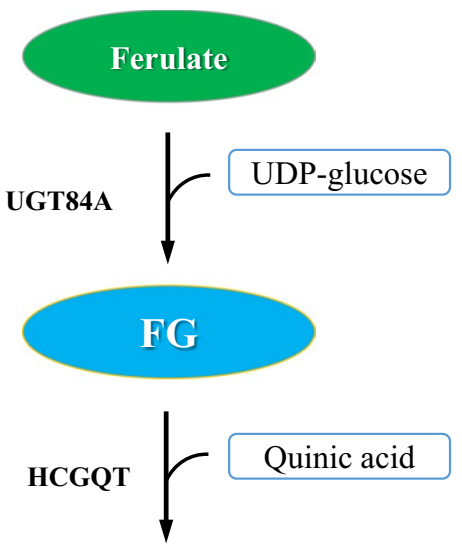

FQA

Fig. 6 The putative biosynthetic pathway of FQA in maize. a The formation of FQA after adding FG into the crude extractive protein from B73 plant. b The formation of FQA after adding FG into the crude extractive protein from bm5-504J mutant. c The putative FQA biosynthetic pathway in maize. FQA, feruloyl quinic acid. FG, feruloyl glucoside. HCGQT, hydroxycinnamoyl D-glucose: quinate hydroxycinnamoyl transferase
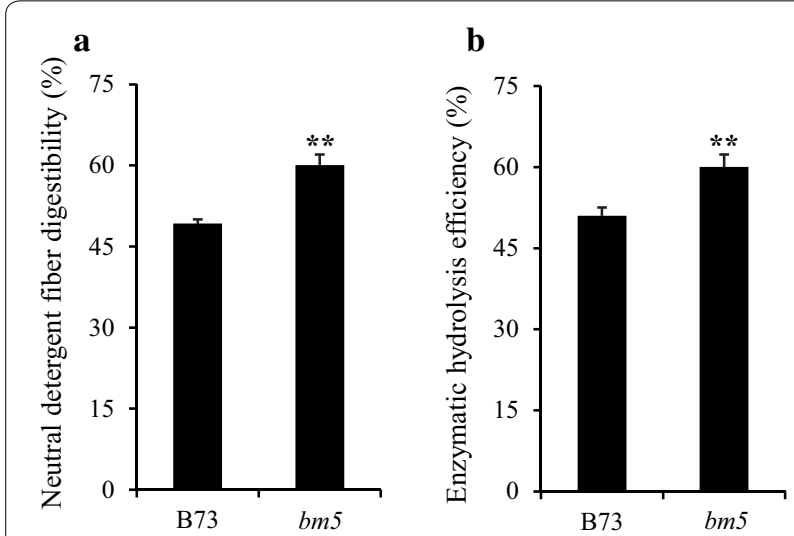

Fig. 7 Effects of bm5 mutation on cell wall digestibility. a Forage digestibility of cell walls of $b m 5$ mutant and $B 73$ wild-type maize. b Saccharification efficiency of cell walls of bm5 mutant and B73 wild-type maize. Stalk samples were collected from 90-day-old bm5-504J mutant and B73 wild-type plants. Values are mean \pm SE $(n=3)$. One or two asterisks indicate significance corresponding to $p<0.05$ or 0.01 (Student's $t$ test)

and $b m 5-505 \mathrm{~J}$ might have a negative influence on the gene expression and/or trigger a rapid mRNA decay in maize. The function of introns in Zm4CL1 is currently unclear, but it deserves further investigation. The other is Ac transposon insertion in the first exon of Zm4CL1 of $b m 5-504 \mathrm{~J}$, which caused a premature stop codon in the transcribed $Z m 4 C L 1$. The shortage of $4 \mathrm{CL}$ activities was observed in crude protein extracts from midribs of the bm5-504J mutant, suggesting that the Ac transposon insertion can significantly reduce the activity of Zm4CL1 in maize. In addition, the insertion of Ac transposon at the first exon of $Z m 4 C L 1$ may produce two truncated proteins as compared with a normal 555 aa Zm4CL1. The shorter version (Zm4CL1-S) remains only the $\mathrm{N}$ terminal 95 aa; The longer one (Zm4CL1-L) could be translated from the first ATG following the insertion sequence and produces a 469 aa protein lacking the partial AMP-binding domain at the $\mathrm{N}$ terminal. We further expressed the two nucleic acid sequences in $E$. coli. The enzyme activity assay of the soluble protein extracts containing the truncated $\mathrm{Zm} 4 \mathrm{CL} 1$ mutants showed that the two truncated versions lost the 4CL function totally. These results demonstrate that the Ac transposon insertion at the first exon accounted for the reduction of $4 \mathrm{CL}$ activity in the crude protein extracted from $b m 5-504 \mathrm{~J}$ mutant.

The impact of $b m 5$ mutation on maize lignin had been investigated by Méchin et al. (2014) [21]. Consistent with the previous study, our lignin analysis data suggest that the biosynthesis of $\mathrm{G}$ lignin was dramatically impaired in the $b m 5$ mutants, whereas the $\mathrm{H}$ lignin was significantly increased. Although the content of S lignin was little affected in $b m 5$ mutant, the relative percentage of $\mathrm{S}$ lignin was still higher than that of B73 wild type. The previous study also revealed a significant reduction in Klason lignin content [21]. However, no difference between bm5 mutant and B73 wild type was observed in our work. 
One possible explanation for the observed effect on the total lignin content is that we used midribs rather than mature stems for lignin analysis besides different methods employed for the measurement of total lignin content. In addition, the content of $\mathrm{H}$ lignin was significantly increased in bm5 mutants, which could partially compensate for the reduction of $\mathrm{G}$ lignin. The previous study has suggested that at least four $4 C L$ paralogous exist in sorghum genome, and the expression levels of these $4 C L$ s including BMR2 are elevated in sorghum Bmr2 mutants [18]. Our results also showed that the expression of Zm4CL2 (GRMZM2G174732) were increased two-to-four-fold in the two $b m 5$ mutants. In particularly, the loss function of $b m 5-504 \mathrm{~J}$ mutation raised the expression levels of the other Zm4CLs significantly. Therefore, we suspected that other 4CLs could compensate for the shortage of $4 \mathrm{CL}$ activity against $p$-coumarate in $b m 5$ mutants, leading to an increase in $\mathrm{H}$ lignin content, since $p$-coumarate rather than caffeate and ferulate is the preferred substrate of the 4CLs [18].

Monolignols are synthesized through a complexity metabolic grid linked to the phenylpropanoid biosynthetic pathway in plants. Therefore, the disruption of lignin biosynthesis has a possibility to shunt its intermediates towards some important soluble phenolic metabolites in plants. A 2.7-5.0-fold increase in FQA was found in the $b m 5$ mutants. FQA is a valuable antioxidant compound for plants, animals, and human. The previous studies have suggested that feruloyl-CoA is the activated intermediate in Solanaceae species, whereas FG is the activated one in other species [24]. Abundant FQA accumulates in maize, which can accumulate in the thrip-resistant cultivars of tomato or be induced in infected maize $[25,26]$. The biosynthetic pathway of FQA, however, still remains largely elusive in this species. FG was a novel phenolic compound present in the $b m 5$ mutants, implying that a biosynthetic pathway exists in maize through FG towards FQA. Moreover, we isolated two UDP-glucoside transferase genes, ZmUGT84A-1 and ZmUGT84A-2, which were involved in the glycosylation of ferulate in vitro (Additional file 11: Fig. S5c, d). Taken together, our results suggest that the $b m 5$ mutation can redirect the redundant ferulate towards FQA through FG biosynthetic pathway.

Disruption of Zm4CL1 significant reduced ADL and, therefore, improves forage digestibility in the $b m 5$ mutant. Consistently, cell wall enzymatic hydrolysis assay indicates that the mutation of $Z m 4 C L 1$ can increase saccharification efficiency of cell wall polysaccharides significantly as well. Although $Z m 4 C L 1$ mutation impaired lignin biosynthesis, the $\mathrm{AcBr}$ lignin content was not changed. It is not surprising, because the $\mathrm{AcBr}$ method reveals more lignin and its derivatives than the $\mathrm{ADL}$ procedure [27]. In addition, the alteration of lignin composition did not affect the contents of cellulose and hemicellulose, suggesting a potential value of the $b m 5$ mutants for lignocellulosic biomass utilization.

\section{Conclusion}

The spontaneous $b m$ mutations can provide useful resources for commercial utilization of corn stover. We characterized $Z m 4 C L 1$ as the mutation gene of maize $b m 5$. Disruption of Zm4CL1 impaired lignin biosynthesis in $b m 5$ mutants and improved the cell wall hydrolysis efficiency. Like $b m 3$, the $b m 5$ mutants will probably to be of interest to be candidates for breeders in the future.

\section{Methods}

Plant materials and growth conditions

The maize stocks, 504I (bm5-PI251930), 504J (bm5PI262480), and 505J (bm5-PI251893) containing the bm5 alleles, were obtained from the Maize Genetics COOP Stock Center. The bm5 NILs developed following a six backcrosses of 504I, 504J, and 505J with B73, respectively, were used for characterization of $b m 5$ gene. The bm5504I and $b m 5$-504J NILs were employed for further phenotypic, molecular, and biochemical analysis. The maize plants were grown in the greenhouse at $26^{\circ} \mathrm{C}$ with $16 \mathrm{~h}$ light $\left(390 \mu \mathrm{E} \mathrm{m}^{-2} \mathrm{~S}^{-1}\right)$.

\section{Genetic analysis and MutMap-assisted gene mapping}

Genomic DNA was extracted from $b m 5-504$ J by the $2 \times$ CTAB method [28]. To confirm the mutant loci, 12 available SSR markers designed in bin 5.04 region were randomly selected and downloaded from MaizeGDB database (Additional file 1: Table S1). Among them, one SSR marker (p-umc1591) was linked with the $b m 5$ phenotype and used to confirm the previous mapping region and identify the homozygous progeny (Additional file 1: Table S1).

The homozygous progeny from $b m 5-504 \mathrm{~J}$ stock was directly crossed with $\mathrm{B} 73$ to generate F1, followed by selfing F1 individuals to generate F2 progeny. DNAs from 96 F2 individuals with the typical brownish midrib phenotype were pooled in an equal ratio and sequenced by Illumina sequencing with depth of more than $10 \times$ coverage. The short reads were then aligned to the reference B73 genome sequences. The single-nucleotide polymorphism markers were analyzed and the SNP-index for each SNP was calculated to quantify the nucleotides difference from the reference B73 genome sequence. The genomic region with SNP-index $=1$ corresponds to the potential mutant position of the $b m 5$. 


\section{Cloning and expression analysis of $Z m 4 C L 1$}

Zm4CL1 genomic sequences from bm5 mutants (504I, 504J, and 505J) and B73 wild-type plants were isolated and sequenced (Additional file 4: Table S3). The primer pairs $\mathrm{S} 1-\mathrm{F}+\mathrm{R}$ and $\mathrm{S} 2-\mathrm{F}+\mathrm{R}$ were designed for rapid detection of the insertion sequences in the mutants (Fig. 2c, Additional file 4: Table S3). Midribs of the second-to-fifth leaves from the top were collected from 60-d old $b m 5$ mutants and B73 wild-type plants. Total RNAs were extracted from the above midribs, and expression levels of $Z m 4 C L 1$ were quantified by RT-PCR and qRTPCR as described by Tang et al. (2014) [16]. The primer pairs for RT-PCR and qRT-PCR were designed around the $3^{\prime}$ untranslated region of Zm4CL1 (Additional file 4: Table S3). To study the influence of the insertions on mature mRNAs, the cDNA fragments of Zm4CL1 were amplified by RT-PCR from $b m 5-504 \mathrm{I}$ and $b m 5-504 \mathrm{~J}$ mutants with the primer pair S1-F $+\mathrm{R}$ (Additional file 4: Table S3). To determine the expression levels of other $Z m 4 C L s$, the qRT-PCR primer pairs were designed in their $3^{\prime}$ untranslated region (Additional file 4: Table S3).

\section{Phylogenetic analysis of 4CLs}

All 4CL protein sequences were downloaded from Phytozome database (http://www.phytozome.net), and the alignments were carried out using CLUSTAL_X [29]. The phylogenetic tree was built using the neighbor-joining method in MEGA 5.0 [30]. Bootstrap values were calculated with 1000 iterations and the values under $70 \%$ were cut off.

\section{CL enzyme activity assay of maize crude extractive protein}

Midribs of the second-to-fifth leaves from the top were collected from 60-day-old bm5 mutants and B73 wildtype plants. Powdered fresh midribs $(\sim 500 \mathrm{mg})$ were extracted for $3 \mathrm{~h}$ at $4{ }^{\circ} \mathrm{C}$ in protein extraction buffer [31]. The samples were centrifuged at $17,900 \times g$ for $20 \mathrm{~min}$ at $4{ }^{\circ} \mathrm{C}$, and the extracts were desalted on PD-10 columns (Pharmacia) and used for 4CL enzyme activity assay against $p$-coumarate, caffeate, and ferulate as described by Liu et al. [31].

\section{Enzyme activity assay of soluble protein extracts of Zm4CL1 proteins}

The coding region of $Z m 4 C L 1$ was amplified from cDNAs of $b m 5$ mutants and B73 wild-type plants using the primers in Additional file 4: Table S3. Zm4CL1-504J would be translated in two versions, Zm4CL1-S and $\mathrm{Zm}$ 4CL1-L, resulted from the Ac transposon insertion in the first exon. The PCR products of Zm4CL1-S, Zm4CL1$L$, and $Z m 4 C L 1$ were subcloned into the pET32a vector (Additional file 4: Table S3). The constructs were introduced into Rosetta E. coli cells for recombinant protein expression [32]. The 4CL enzyme activities against $p$-coumarate, caffeate, and ferulate were determined as describe by Liu et al. [31].

\section{Microarray analysis}

The midribs were separated from the leaves of 60-dayold bm5-504J mutants and B73 wild-type plants. RNA extraction and purification, probe labeling, hybridization, and scanning for Affymetrix microarray analysis were conducted as previously described [4].

\section{Lignin content and composition analysis}

Midribs of the fourth-to-fifth leaves from the top were collected from 60-day-old bm5 mutants and B73 wildtype plants. Soluble extracts were removed from the ground lyophilized samples by four successive extractions with chloroform/methanol $(2: 1 \mathrm{v} / \mathrm{v})$, methanol, methanol $/ \mathrm{H}_{2} \mathrm{O}(1: 1 \mathrm{v} / \mathrm{v})$, and water at room temperature as described by Chen and Dixon [1], and the remaining CWRs were lyophilized for lignin analysis. The quantification of lignin content was conducted by acetyl bromide method [33]. The lignin compositions were measured by thioacidolysis method $[34,35]$.

\section{Profiling analysis of soluble phenolics}

Midribs of the second-to-fifth leaves from the top were collected from 60-day-old bm2-ref mutants and B73 wild-type plants and homogenized in liquid nitrogen and lyophilized. The methanolic extracts from lyophilized materials including B73 wild type, bm5-504I and bm5504J mutants, were subjected to soluble phenolic profiling analysis by LC-PDA-ESI-MS/MS [36]. The phenolic compounds were identified based on their UV-visible spectra, mass spectra, and comparison with the authentic standard compound and the MS data reported by Eloy et al. [37]. The authentic standard compounds, $p$-coumarate, caffeate, and ferulate, were ordered from SigmaAldrich (St. Louis, MO, USA). The FG was prepared using the production of the identified AtUGT84A1 toward ferulate and UDP-glucose [38]. Moreover, the AtUGT84A1 homologous genes, ZmUGT84A-1 and ZmUGT84A-2 isolated from B73 wild type, were cloned into pET32a vector, respectively, to produce recombinant proteins in E. coli [32]. Primers for gene cloning and vector construction were listed in the Additional file 4: Table S3. The soluble protein extracts containing ZmUGT84A-1 and ZmUGT84A-2 identified by SDS-PAGE (Additional file 14: Fig. S6) were used to convert ferulate and UDPglucose into FG in vitro [37]. The purified FG through HPLC was further identified by LC-PDA-MS/MS [36]. In addition, FQA was synthesized from FG and quinic acid after incubation with the crude extractive proteins 
from midribs of $b m 5$ mutant as described by Villegas and Kojima (1986) [39].

\section{Cell wall digestibility analysis}

Stalk samples were collected from bm5-504J mutants and B73 wild-type plants at the R1 stage (silk emergence) and dried in an oven at $40{ }^{\circ} \mathrm{C}$ for 1 week. Samples were ground through a Wiley mill with a $1-\mathrm{mm}$ sieve for analysis of neutral detergent fiber (NDF), acid detergent fiber (ADF), and in vitro true dry matter digestibility (IVTDMD) using NIRS [40]. ADL was measured using an ANKOM 200 Fiber Analyzer (ANKOM Technology Corp.) [41]. ADL, NDF, and ADF were used to calculate cellulose (ADF-ADL) and hemicellulose (NDF-ADF) contents. IVNDF, ADF, and IVTDMD were employed for IVNDFD calculation by the formula $(\mathrm{NDF}+\mathrm{IVTDMD}-100) / \mathrm{NDF} \times 100$.

Saccharification of maize stalk samples was performed following the analytical procedure described by the National Renewable Energy Laboratory (LAP-009: Enzymatic Saccharification of Lignocellulosic Biomass). Briefly, solubilized sugars were yielded from CWRs digested by pretreatment with $1.5 \% \mathrm{H}_{2} \mathrm{SO}_{4}$ at $121{ }^{\circ} \mathrm{C}$ for $40 \mathrm{~min}$ and then exposure to a cellulase and cellobiase mixture for $72 \mathrm{~h}$ after washing with Milli-Q water. The solubilized sugars were detected with the phenol-sulfuric acid assay method [42].

\section{Statistical analysis}

Samples were collected from three biological replicates. The mean values were used for statistical analyses. Data from each trait were subjected to Student's $t$ test. The significance of treatments was tested at the $p=0.05$ and 0.01 levels. Standard errors were provided in all tables and figures as appropriate.

\section{Additional files}

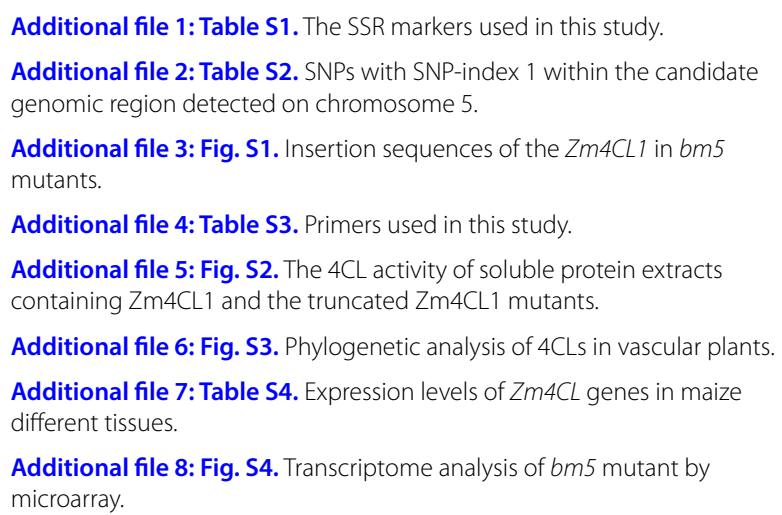

Additional file 9: Table S5. Signal intension of the probe sets of regulated genes in bm5-504J mutant and B73 wild-type plant.

Additional file 10: Table S6. Signal intension of the probe sets of lignin genes in bm5-504J mutants and B73 wild-type plants.

Additional file 11: Fig. S5. Expression levels of other $4 C L$ paralogs in bm5 mutants.

Additional file 12: Table S7. Lignin content and composition of bm5 mutant.

Additional file 13: Table S8. LC-PDA-ESI-MS/MS identification of soluble phenolics in methanolic extracts from midribs of the bm5 mutant.

Additional file 14: Fig. S6. SDS-PAGE analysis of recombinant AtUGT84A1, ZmUGT84A-1, and UGT84A-2 proteins.

Additional file 15: Fig. S7. The characterization of UDP-glucoside transferase forming glucose ester with ferulate in vitro.

Additional file 16: Fig. S8. The biosynthesis and characterization of FG and FQA in vitro.

Additional file 17: Fig. S9. UV-visible spectra of the remarkably accumulated products formed after adding FG into the crude extractive proteins from B73 and bm5-504J mutant.

Additional file 18: Table S9. ADL, hemicellulose, and cellulose contents of bm5 mutants and B73 wild-type plants.

\section{Abbreviations}

AcBr: acetyl bromide; ADF: acid detergent fiber; ADL: acid detergent lignin; bm: brown midrib; CAD: cinnamyl alcohol dehydrogenase; 4CL: 4-coumarate: COA ligase; COMT: caffeic acid O-methyltransferase; CWR: cell wall residue; $F G$ : feruloyl glycoside; FQA: feruloyl quinic acid (FQA); G: guaiacyl; IVTDMD: in vitro true dry matter digestibility; LC-PDA-ESI-MS: liquid chromatography coupled with photo-diode array detection and electrospray ionization tandem mass spectrometry; NDF: neutral detergent fiber; NDFD: neutral detergent fiber digestibility; NIL: near-isogenic lines; NIRS: near infrared reflectance spectroscopy; PCR: polymerase chain reaction; RT-PCR: reverse transcription polymerase chain reaction; qRT-PCR: quantitative transcription polymerase chain reaction; S: syringyl; SSR: simple sequence repeat.

\section{Authors' contributions}

$C F, W X$, and $Z W$ designed the research. $W X, Z W, Y L, Y L, K S, Z B, S G, Z H, Z Z, Y B$, $J S$, and GY performed the experiments. CF, WX, ZW, SG, and ZH analyzed the data. CF and WX wrote the article. All authors read and approved the final manuscript.

\section{Author details}

${ }^{1}$ Key Laboratory of Biofuels, Shandong Provincial Key Laboratory of Energy Genetics, Qingdao Institute of Bioenergy and Bioprocess Technology, Chinese Academy of Sciences, Qingdao 266101, China. ${ }^{2}$ Institute of Plant Stress Biology, State Key Laboratory of Cotton Biology, Department of Biology, Henan University, Kaifeng 475001, China. ${ }^{3}$ Maize Research Institute, Sichuan Agricultural University, Chengdu 611130, Sichuan, China. ${ }^{4}$ School of Animal Science and Technology, Qingdao Agricultural University, Qingdao, China.

\section{Acknowledgements}

Not applicable.

\section{Competing interests}

The authors declare that they have no competing interests.

\section{Availability of data and materials}

All data generated or analyzed during this study are included in this published article and its supplementary information files.

\section{Consent for publication}

Not applicable.

Ethics approval and consent to participate Not applicable. 


\section{Funding}

The work was supported by the National Key Technologies Research \& Development Program-Seven Major Crops Breeding Project (No. 2016YFD0101803), the National Natural Science Foundation of China (Nos. 31470390 and 31800254), Major Program of Shandong Province Natural Science Foundation (No. ZR2018ZB0213), Qingdao City: Program for Entrepreneurial and Innovative Leading Talents (No. 16-8-3-15-zhc), and Funds of Shandong Improved Variety Program (2017LZGC005).

\section{Publisher's Note}

Springer Nature remains neutral with regard to jurisdictional claims in published maps and institutional affiliations.

\section{Received: 29 November 2018 Accepted: 29 March 2019} Published online: 10 April 2019

\section{References}

1. Chen F, Dixon RA. Lignin modification improves fermentable sugar yields for biofuel production. Nat Biotechnol. 2007;25:759-61.

2. Boerjan W, Ralph J, Baucher M. Lignin biosynthesis. Annu Rev Plant Biol. 2003;54:519-46.

3. Carroll A, Somerville C. Cellulosic biofuels. Annu Rev Plant Biol. 2009;60:165-82.

4. Tarasov D, Leitch M, Fatehi P. Lignin-carbohydrate complexes: properties, applications, analyses, and methods of extraction: a review. Biotechnol Biofuels. 2018;11:269.

5. Knobloch KH, Hahlbrock K. 4-Coumarate: CoA ligase from cell-suspension cultures of Petroselinum hortense Hoffm: partial purification, substrate specificity and further properties. Arch Biochem Biophys. 1977;184:237-48.

6. Lavhale SG, Kalunke RM, Giri AP. Structural, functional and evolutionary diversity of 4-coumarate-CoA ligase in plants. Planta. 2018:248:1063-78.

7. Li Y, Kim Jl, Pysh L, Chapple C. Four isoforms of Arabidopsis 4-coumarate: CoA ligase have overlapping yet distinct roles in phenylpropanoid metabolism. Plant Physiol. 2015;169:2409-21.

8. Gui J, Shen J, Li L. Functional characterization of evolutionarily divergent 4-coumarate: coenzyme A ligases in rice. Plant Physiol. 2011;157:574-86.

9. Xu B, Escamilla-Trevino LL, Sathitsuksanoh N, Shen Z, Shen H, Zhang YHP, Dixon RA, Zhao B. Silencing of 4-coumarate: coenzyme A ligase in switchgrass leads to reduced lignin content and improved fermentable sugar yields for biofuel production. New Phytol. 2011;192:611-25.

10. Jung JH, Kannan B, Dermawan H, Moxley GW, Altpeter F. Precision breeding for RNAi suppression of a major 4-coumarate: coenzyme A ligase gene improves cell wall saccharification from field grown sugarcane. Plant Mol Biol. 2016;92:505-17.

11. Park J, Yoo C, Flanagan A, Pu Y, Debnath S, Ge X, Ragauskas AJ, Wang Z. Defined tetra-allelic gene disruption of the 4-coumarate: coenzyme A ligase 1 ( $P$ V4CL 1) gene by CRISPR/Cas9 in switchgrass results in lignin reduction and improved sugar release. Biotechnol Biofuels. 2017;10:284

12. Liu T, Yao R, Zhao Y, Xu S, Huang C, Luo J, Kong L. Cloning, functional characterization and site-directed mutagenesis of 4-coumarate: coenzyme A ligase (4CL) involved in coumarin biosynthesis in Peucedanum praeruptorum Dunn. Front Plant Sci. 2017;8:4.

13. Sattler SE, Funnell-Harris DL, Pedersen JF. Brown midrib mutations and their importance to the utilization of maize, sorghum, and pearl millet lignocellulosic tissues. Plant Sci. 2010;178:229-38.

14. Halpin C, Holt K, Chojecki J, Oliver D, Chabbert B, Monties B, Edwards K, Barakate A, Foxon GA. Brown-midrib maize (bm 1) - a mutation affecting the cinnamyl alcohol dehydrogenase gene. Plant J. 1998;14:545-53.

15. Li L, Hill-Skinner S, Liu S, Beuchle D, Tang H, Yeh CT, Nettleton D, Schnable PS. The maize brown midrib4 (bm4) gene encodes a functional folylpolyglutamate synthase. Plant J. 2015;81:493-504.

16. Tang H, Liu S, Hill-Skinner S, Wu W, Reed D, Yeh CT, Nettleton D, Schnable PS. The maize brown midrib2 (bm2) gene encodes a methylenetetrahydrofolate reductase that contributes to lignin accumulation. Plant J. 2014;77:380-92.
17. Vignols F, Rigau J, Torres MA, Capellades M, Puigdomenech P. The brown midrib3 (bm3) mutation in maize occurs in the gene encoding caffeic acid O-methyltransferase. Plant Cell. 1995;7:407-16.

18. Saballos A, Sattler SE, Sanchez E, Foster TP, Xin Z, Kang C, Pedersen JF, Vermerris W. Brown midrib2 (Bmr2) encodes the major 4-coumarate: coenzyme A ligase involved in lignin biosynthesis in sorghum (Sorghum bicolor (L.) Moench). Plant J. 2012;70:818-30.

19. Haney LJ, Hake S, Scott MP. Allelism testing of Maize Coop Stock Center lines containing unknown brown midrib alleles. Maize Genet Cooperat Newsl. 2008;82:4-5.

20. Lawrence CJ, Seigfried TE, Brendel V. The maize genetics and genomics database. The community resource for access to diverse maize data. Plant Physiol. 2005;138:55-8.

21. Mechin V, Laluc A, Legee F, Cezard L, Denoue D, Barriere Y, Lapierre C. Impact of the brown-midrib bm5 mutation on maize lignins. J Agric Food Chem. 2014;62:5102-7.

22. Ali F, Scott P, Bakht J, Chen Y, Lubberstedt T. Identification of novel brown midrib genes in maize by tests of allelism. Plant Breeding. 2010;129:724-6.

23. Rose AB. Intron-mediated regulation of gene expression. In: Reddy ASN, Golovkin M, editors. Nuclear pre-mRNA processing in plants. Springer, Berlin: Heidelberg; 2008. p. 277-90.

24. Niggeweg R, Michael AJ, Martin C. Engineering plants with increased levels of the antioxidant chlorogenic acid. Nat Biotechnol. 2004;22:746-54.

25. Leiss KA, Choi YH, Verpoorte R, Klinkhamer PGL. An overview of NMRbased metabolomics to identify secondary plant compounds involved in host plant resistance. Phytochem Rev. 2011:10:205-16.

26. Balmer D, de Papajewski DV, Planchamp C, Glauser G, Mauch-Mani B. Induced resistance in maize is based on organ-specific defence responses. Plant J. 2013;74:213-25.

27. Fu C, Sunkar R, Zhou C, Shen H, Zhang J, Matts J, Wolf J, Mann DGJ, Stewart CN, Tang Y, Wang Z. Overexpression of miR156 in switchgrass (Panicum virgatum L.) results in various morphological alterations and leads to improved biomass production. Plant Biotechnol J. 2012;10:443-52.

28. Porebski S, Bailey LG, Baum BR. Modification of a CTAB DNA extraction protocol for plants containing high polysaccharide and polyphenol components. Plant Mol Biol Rep. 1997;15:8-15.

29. Thompson JD, Gibson TJ, Plewniak F, Jeanmougin F, Higgins DG. The CLUSTAL_X windows interface: flexible strategies for multiple sequence alignment aided by quality analysis tools. Nucleic Acids Res. 1997;25:4876-82

30. Tamura K, Peterson D, Peterson N, Stecher G, Nei M, Kumar S. MEGA5: molecular evolutionary genetics analysis using maximum likelihood, evolutionary distance, and maximum parsimony methods. Mol Biol Evol. 2011;28:2731-9.

31. Liu J, Shi R, Li Q, Sederoff RR, Chiang VL. A standard reaction condition and a single HPLC separation system are sufficient for estimation of monolignol biosynthetic pathway enzyme activities. Planta. 2012;236:879-85.

32. Xiong W, Fu J, Kollner TG, Chen X, Jia Q, Guo H, Qian P, Guo H, Wu G, Chen F. Biochemical characterization of microbial type terpene synthases in two closely related species of hornworts, Anthoceros punctatus and Anthoceros agrestis. Phytochemistry. 2018;149:116-22.

33. Hatfield RD, Grabber J, Ralph J, Brei K. Using the acetyl bromide assay to determine lignin concentrations in herbaceous plants: some cautionary notes. J Agr Food Chem. 1999;47:628-32.

34. Lapierre C, Pollet B, Rolando C. New insights into the molecular architecture of hardwood lignins by chemical degradative methods. Res Chem Intermediat. 1995;21:397-412.

35. Lapierre C, Monties B, Rolando C. Thioacidolysis of poplar lignins-identification of monomeric syringyl products and characterization of guaiacylsyringyl lignin fractions. Holzforschung. 1986;40:113-8.

36. Zhang JY, de Carvalho MHC, Torres-Jerez I, Kang Y, Allen SN, Huhman DV, Tang YH, Murray J, Sumner LW, Udvardi MK. Global reprogramming of transcription and metabolism in Medicago truncatula during progressive drought and after rewatering. Plant Cell Environ. 2014;37:2553-76.

37. Eloy NB, Voorend W, Lan W, Saleme ML, Cesarino I, Vanholme R, Smith RA, Goeminne G, Pallidis A, Morreel K, Nicomedes J Jr, Ralph J, Boerjan W. Silencing CHALCONE SYNTHASE in maize impedes the incorporation of tricin into lignin and increases lignin content. Plant Physiol. 2017;173:998-1016. 
38. Lim EK, LiY, Parr A, Jackson R, Ashford DA, Bowles DJ. Identification of glucosyltransferase genes involved in sinapate metabolism and lignin synthesis in Arabidopsis. J Biol Chem. 2001;276:4344-9.

39. Villegas RJA, Kojima M. Purification and characterisation of hydroxycinnamoyl D-glucose quinate hydroxycinnamoyl transferase in the root of sweet potato, I pomoea batatas LAM. J Biol Chem. 1986;236:8729-33.

40. Krizsan SJ, Nyholm L, Nousiainen J, Südekum KH, Huhtanen P. Comparison of in vitro and in situ methods in evaluation of forage digestibility in ruminants. J Anim Sci. 2012;90:3162-73.
41. Lorenz AJ, Anex RP, Isci A, Coors JG, de Leon N, Weimer PJ. Forage quality and composition measurements as predictors of ethanol yield from maize (Zea mays L.) stover. Biotechnol Biofuels. 2009;2:5.

42. Dubois M, Gilles KA, Hamilton JK, Rebers PA, Smith F. Colorimetric method for determination of sugars and related substances. Anal Chem. 1956;28:350-6
Ready to submit your research? Choose BMC and benefit from:

- fast, convenient online submission

- thorough peer review by experienced researchers in your field

- rapid publication on acceptance

- support for research data, including large and complex data types

- gold Open Access which fosters wider collaboration and increased citations

- maximum visibility for your research: over $100 \mathrm{M}$ website views per year

At BMC, research is always in progress.

Learn more biomedcentral.com/submissions 